ISSN: 2689-2707

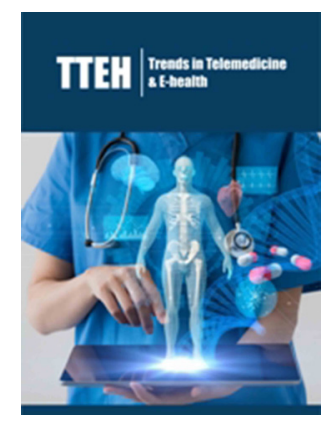

*Corresponding author: Georgekutty Kuriala Kochuchakkalackal, The Graduate School, Philippines

Submission: 戝 June 17, 2019

Published: 悳June 24, 2019

Volume 1 - Issue 4

How to cite this article: Georgekutty Kuriala Kochuchakkalackal. Telemedicine: A New Perspective in the Treatment of Internet Gaming Disorder Among Adolescents. Trends Telemed E-Health. 1(4). TTEH. 000518. 2019. DOI: 10.31031/TTEH.2019.01.000518

Copyright@ Georgekutty Kuriala Kochuchakkalackal, This article is distributed under the terms of the Creative Commons Attribution 4.0 International License, which permits unrestricted use and redistribution provided that the original author and source are credited.

\section{Telemedicine: A New Perspective in the Treatment of Internet Gaming Disorder Among Adolescents}

\author{
Georgekutty Kuriala Kochuchakkalackal ${ }^{1,2 *}$ \\ ${ }^{1}$ The Graduate School, University of Santo Tomas, Manila, Philippines \\ ${ }^{2}$ College of Medicine, Emilio Aguinaldo College, Manila, Philippines
}

\section{Opinion}

The internet era brings an enormous opportunity to contribute in the universal goal of providing quality clinical and health care especially addressing emerging issues that significantly affect the mental health of adolescents brought about by modern technology. What used to be unimaginable is made possible by the integration of advancement in information technology, electronic communications technology, telecommunications, computers and mobile technology with the field of medicine giving rise to the inception of the field of telemedicine as early as the 1960s using radio and telephone [1]. Presently, with the emergence of new health conditions needing immediate diagnosis and treatment, the internet facility plays an important role in communication and in providing these services to patients real time. The World Health Organization (WHO) has recently recognized gaming disorder as a mental health condition as it continues to affect adolescents in increasing number of countries.

Empirical evidences associating Internet Gaming Disorder (IGD) with poor psychological well-being is also increasing and, in severe cases, death occurs. We are challenging the medical profession, both the professionals and the health care service providers, to expand the practice of telemedicine as a new perspective in the treatment of adolescents with gaming disorder in consideration of patient well-being, its negative to severe consequences in daily life, and its mission to help ensure patients are provided with best health care service available [2]. Telemedicine uses electronic communications media such as tele-conferencing, imagesharing, document sharing, and mobile technology. Depending on the patient's geographical location and health care needs, a patient avails (real time) interactive services, remote patient monitoring and store and forward benefits of telemedicine. Real time interactive services enable the patient, doctor and/or medical staff to be in communication with each other over computer or mobile phone at any given time. Remote patient monitoring allows patients to be monitored outside of the conventional clinical setting. Store and forward is a telecommunications technique when there is intermittent network connectivity usually experienced in the wilderness or environments requiring high mobility [3]. Information is temporarily stored then forwarded to its destination at a later time. To date, the implementation of telemedicine has expanded to medical fields such as dermatology, cardiology and behavioral health to provide clinical and specialty care to those without access to physicians or hospitals, or both. The sophistication in technologies gave rise to telerehabilitation, telenursing, tele neuropsychology, telepsychiatry, teleradiology, telepathology, teledentistry among others.

The advantages of adopting telemedicine includes:

A. Overcoming distance barrier attributed to the global reach or accessibility of the internet,

B. Reducing or totally eliminating travel distance and time for consultation and treatment, 
C. Real time or immediate availability of health care for those with equally scarce and inaccessible resources,

D. Opening option for patients to choose rehabilitation at home,

E. Based on the foregoing, proves to be less costly and more convenient for both patient and doctor, and

F. Records management promoting continuity and coordination of care by virtue of available comprehensive information.

It is noteworthy that it was also observed by clinicians that the practice of telemedicine

A. Encourages healthier behavior among patients and

B. Empowers patients to manage their conditions [4]. Telemedicine can now be more beneficial to provide quality service as internet and modern equipments allow for better voice or audio quality, clearer video or imaging, and faster connectivity and communication. With e-health, it is able to furnish specialized medical information and health education as well as enable and encourage online group discussions for peer-to-peer support.

Over the years, many studies have linked Internet Gaming Disorder (IGD) with poor psychological well-being. Its other negative consequences include impaired daily functioning and self-harming behaviors. One of the nine symptoms of IGD defined by the American Psychiatric Association in the latest 2013 update of the Diagnostic and Statistical Manual $5^{\text {th }}$ edition (DSM-5) is that adolescents deceive family members, therapists or others on the amount of time spent gaming aside from loss of interest in previous hobbies, withdrawal symptoms and continuous gaming despite knowledge of its negative consequences etc. that lead to a significant impairment or clinical distress.

For this reason, adolescents who are at risk of IGD will substantially benefit from the practice of telemedicine as it encourages the young to seek immediate professional help. Online consultation will alleviate the surrounding social stigma among this age bracket in having to visit a doctor and divulge details of the experienced disorder. In this current scenario, telemedicine and e-health significantly complement each other in addressing IGD by promoting efficiency when immediate health care is required, mitigating compulsive internet gaming from turning into fullblown addiction, and educating patients of health information. Studies indicate that adolescents with IGD and psychological issues are more confident online than offline. They prefer to be isolated, are more verbose or are able to communicate using their devices. Despite the many benefits that can be gained from telemedicine, it is not without limitations. Licensing and regulatory requirements such as data privacy that are different from every country and its states put a limitation on its implementation worldwide. In the absence of global standards, the quality of health care will still differ from each area. The absence of physical interaction among the patient, doctor and/or medical practitioners is another limitation in its practice. Nevertheless, the benefits of telemedicine outweigh its limitations [5]. It is still perceived that the field of telemedicine has the most potential in successfully addressing the emerging issue of IGD among the affected adolescents regardless of geography and in providing equal care to those without access to health care locally. As there is yet to be established an effective intervention program for IGD among adolescents in a clinical setting, it is a challenge for telemedicine to explore and develop an efficacious remedy or treatment program that is also culturefree in reducing the symptoms or risk of IGD and improving the psychological well-being of adolescents.

\section{References}

1. Bashshur RL (2002) Telemedicine and healthcare. Telemedicine Journal and e-health 8 (1).

2. Eastwood B (2012) 10 ways telemedicine is changing healthcare IT.

3. (2010) Telemedicine opportunities and developments in member states. World Health Organization, Switzerland.

4. (2013) What is telemedicine? American Telemedicine Association, USA.

5. Hjem NM (2005) Benefits and drawbacks of telemedicine. Journal of telemedicine and telecare 11(2): 60-70. 